\title{
La Rsi di fronte a sfollati, profughi ed evacuati
}

Rovatti

ISSN: 2282-4979

DOI: 10.12977/ere-

view263

Dopo il settembre 1943 allo sfollamento causato dai bombardamenti si unirono nuovi movimenti di popolazione determinati dalla fuga volontaria dalle zone di combattimento, dalla ritirata verso nord di fascisti con le loro famiglie e dalle evacuazioni ordinate dai tedeschi per motivi militari. Alcuni capi provincia, dovettero organizzare punti di raccolta per gli evacuati lungo le rotte che portavano a nord. Le province settentrionali furono incaricate di assorbire quote crescenti di profughi ed evacuati.

Since September 1943 the evacuation caused by the bombing was combined with new population movements determined by voluntary escape from the combat zones, voluntary flight of Fascist family groups and the evacuations ordered by the German authorities for military reasons. Some 'province chiefs' (the prefects of the Rsi), had to organise assembly points for evacuees along the routes leading to the north. The northern provinces were thus instructed to absorb increasing quotas of refugees and evacuees.

\section{Premessa}

È importante, prima di tutto, definire i termini "sfollati", "profughi" ed "evacuati" che corrispondono a tre gruppi di italiani che, durante il secondo conflitto mondiale, si spostarono all'interno della penisola a causa della guerra. Per il regime fascista «sfollati» erano coloro che si allontanavano dalle città colpite o minacciate dai bombardamenti; «profughi» venivano chiamati quelli che risalivano la penisola per fuggire all'avvicinarsi del fronte oppure si erano trovati, per vari motivi, lontani dai territori nei quali risiedevano ormai già occupati dagli Alleati 
(si noti che il termine "profughi" richiama quello di "profughi politici" e probabilmente il regime li considerava tali, poiché riteneva che molti si muovessero per sfuggire al nemico); «evacuati» erano, invece, i tanti che dovettero obbligatoriamente lasciare le loro case in seguito a perentori ordini dati dalle forze armate italiane (nei primi giorni del giugno 1940 e nel luglio 1943) o tedesche (dall'ottobre 1943 al febbraio 1945). Dall'autunno del 1943 non fu sempre possibile fare una distinzione poiché i tre gruppi andarono confondendosi e mischiandosi; a volte, quindi, il regime li raccolse sotto l'unico termine «sfollati». Si sta parlando di centinaia di migliaia di persone che, a partire dalla prima notte di guerra (10-11 giugno 1940), coi primi bombardamenti, abbandonarono le loro case e tutto, o quasi, ciò che possedevano.

\section{Una sfida importante per il consenso}

L'importanza del fenomeno dello sfollamento nell'esperienza degli italiani in guerra non è dovuta solo alla sua estensione quantitativa e geografica, ma anche all'estrema influenza che esso ebbe sul consenso al fascismo. La creazione nel 1930 del Comitato centrale interministeriale per la protezione antiaerea (Ccipaa), che aveva il compito di porre le basi organizzative per la costituzione di un sistema di difesa dei civili dai bombardamenti, dimostra che già allora lo Stato fascista era consapevole del grande peso che avrebbe avuto proteggere gli italiani dalla guerra aerea, e, poiché lo sfollamento apparve subito essere la strada migliore, anzi l'unica in tal senso, era chiaro che la sua gestione sarebbe stata una partita importante. Forse a quei tempi le preoccupazioni maggiori erano quelle di salvaguardare la popolazione e i lavoratori delle industrie che producevano per il conflitto, ma posso dire con sicurezza che dall'autunno del 1942 a queste si affiancò, divenendo sempre più rilevante, quella per la tenuta del consenso al regime e alla "sua" guerra.

Nel corso dell'intera guerra l'organizzazione dello sfollamento e dell'assistenza agli sfollati fu nelle mani del ministero dell'Interno e degli enti pubblici locali (prefetture e municipi), ma il peso che aveva sul mantenimento del consenso e sulla tenuta del fronte interno era ben chiaro a tutte le anime del regime. I civili erano colpiti su larga scala direttamente dalla guerra militare (addirittura erano considerati strategicamente bersagli di essa): proteggerli e assisterli era fondamentale per la tenuta del fronte interno, e anche per quella del fronte esterno poiché i soldati non avrebbero accettato che il loro governo lasciasse le loro famiglie in pericolo, abbandonate a loro stesse. Se non si era in grado di proteggere i civili dagli attacchi aerei, bisognava almeno assisterli nella loro fuga e nella 
loro ricerca di un luogo più sicuro, e anche vestirli e sfamarli dopo che avevano abbandonato o perso tutto sotto le bombe.

E che gli abitanti delle città italiane non potessero essere protetti dai bombardamenti il regime lo sapeva dal 1931 quando il Ccipaa si era immediatamente reso conto dell'estrema difficoltà, dovuta a motivi logistici, economici e psicologici, di garantire nelle città più grandi forme di difesa della popolazione civile realmente efficaci e aveva così affermato che il provvedimento più sicuro sarebbe stato quello di diminuire drasticamente la popolazione da proteggere [Adorno 1986, 281, 297 nota 1].

Alle soglie dell'entrata in guerra i centri urbani erano attrezzati per proteggere solo coloro che non dovevano allontanarsi da essi o perché dovevano salvaguardarne le strutture vitali e i servizi essenziali (ad esempio l'acquedotto) e limitarne la distruzione (per esempio gli uomini della protezione antiaerea e i pompieri), o perché dovevano combattere lo sciacallaggio (polizia e carabinieri). Nel 1939, di conseguenza, i rifugi pronti in caso di una guerra (tutti rifugi pubblici) erano sufficienti a ospitare solo lo $0,71 \%$ della popolazione italiana, il $2 \%$ degli abitanti dei capoluoghi di provincia. A guerra in corso un po' alla volta furono creati altri rifugi pubblici, ma soprattutto si puntò a costringere i proprietari di stabili a crearne di privati puntellando con assi di legno e rinforzando con sacchi di sabbia le cantine. Una soluzione non sufficiente, né efficace sia perché tali proprietari, quando non ignorarono del tutto questa direttiva, cercarono di spendere il meno possibile, sia perché molte cantine erano di per sé inadatte a ospitare rifugi perché non avevano una doppia uscita e spesso ospitavano caldaie o tubature del gas. A queste carenze della difesa passiva si univano quelle della difesa attiva, soprattutto della contraerea: povera in uomini, in mezzi e, soprattutto, nella qualità dei mezzi [Gioannini e Massobrio 2007].

Nonostante la drastica affermazione fatta dal Ccipaa nel 1931, il "piano di sfollamento" che fu predisposto con successivi provvedimenti (e correzioni di essi) tra quell'anno e il 1939 e che coinvolgeva 55 capoluoghi di provincia puntò sulla promozione dello sfollamento volontario di coloro che non erano necessari alla vita essenziale delle città, al mantenimento dell'ordine, alla produzione bellica e alla protezione antiaerea, e non sull'organizzazione e gestione di uno sfollamento obbligatorio. L'intera "faccenda" era affidata alle autorità locali: il coordinamento e il controllo spettavano alle prefetture, gli aspetti logistici e assistenziali (cioè il vero intervento quotidiano, anche economico) ai comuni, soprattutto agli Enti comunali di assistenza (Eca). Sfollare dove, come? Su questi punti le direttive lasciavano molto spazio all'improvvisazione e quando il Piano di diradamento della popolazione civile scattò automaticamente in tutti quei 55 capoluoghi nella 
notte del 10 giugno del 1940, nel giro di dieci giorni la situazione diventò ingestibile al punto che il ministero dell'Interno fu costretto a ordinare non solo di interrompere completamente il Piano di diradamento, ma anche di invertirne il flusso:

Occorre assolutamente contenere sfollamento volontario [...] Prefetti provincie interessate facciano pertanto opera persuasione in tal senso cominciando col negare ogni facilitazione di viaggio alt Est anzi opportuno che sfollati volontari siano consigliati rientrare loro normale residenza alt Per quanto riferiscesi sfollati indigenti, Prefetti interessati provvedano loro mezzi rimpatrio, avvertendoli che, in caso di rifiuto, verrà loro cessare, a datare domenica ventura, ogni forma assistenza. Raccomando massimo impegno et massimo tatto'.

Era una dichiarazione di totale insuccesso nella gestione di quell'iniziale sfollamento ed era anche la completa disfatta di quell'unica soluzione che il Ccipaa aveva individuato nel 1931 per difendere la popolazione civile.

Tra il 20 giugno 1940 e la fine del 1941 le disposizioni centrali riguardanti lo sfollamento furono poche e poco significative. Non fu elaborato, e nemmeno definito un po' alla volta con circolari consecutive, un qualche piano di organizzazione, gestione e coordinamento dall'alto della fuga dalle città bombardate $\mathrm{e}$ delle conseguenze di questa. Mentre quella fuga, nonostante i tentativi del regime di frenarla, non solo era proseguita, ma era anche andata intensificandosi, sia perché gli attacchi aerei sulle maggiori città del nord e del sud non erano cessati, sia - ritengo soprattutto - perché l'iniziale disponibilità della maggior parte degli italiani a sopportare i sacrifici imposti dalla guerra aerea si era progressivamente incrinata di fronte al fatto che il conflitto si stava prolungando molto più del previsto. Con la capitolazione della Francia, infatti, la guerra era sembrata davvero un "problema" di ancora poche settimane. Ma le settimane erano diventate mesi e a poco a poco la fiducia e le speranze si erano trasformate in inquietudine e nel timore che il conflitto sarebbe durato ancora a lungo, «mentre - scrivono Gioannini e Massobrio - nel frattempo non migliora[va] la capacità del regime di difendere le vite e le proprietà dei cittadini» [Gioannini e Massobrio 2007, 13]. Riuscire a proteggere la vita dei civili (che erano anche le famiglie dei soldati al fronte) era dunque una partita più che importante per il regime e se non si era

Telegramma n. 46750 inviato il 20.06.1940 dal Gabinetto del Ministero dell'Interno ai prefetti e, p.c., alla segreteria del Pnf, allo Stato maggiore dell'esercito, al Comando gruppo armate ovest, ai comandi della la e della $4^{\mathrm{a}}$ Armata, al Comando generale dei Carabinieri, al Comando generale della Guardia di finanza, alla Presidenza generale della Croce rossa, ai Comandi di difesa territoriale di Torino, Genova, Alessandria e Milano, alla Direzione generale protezione antiaerea, alla Presidenza del Consiglio dei ministri e alla Direzione generale di pubblica sicurezza, Archivio centrale dello Stato (d'ora in poi Acs), Ministero dell'Interno (d'ora in poi MI), Direzione generale pubblica sicurezza (d'ora in poi Dgps), Divisione affari generali e riservati (d'ora in poi Agr), cp, A5Gllgm, f. 26, b. 60, sf. 1 Ag, ins. 1 Ag1; 0 anche Acs, MI, Direzione generale servizi di guerra (d'ora in poi Dgsg), b. 8. 
in grado di farlo nelle città bisognava almeno tentare di gestire meglio lo sfollamento. Dopo una ristrutturazione degli uffici centrali il 5 maggio 1941, con la nascita della Direzione generale per i servizi di protezione antiaerea (Dgspa) e dell'Ispettorato per i servizi di guerra (Isg), quest'ultimo iniziò a porre le basi per una organizzazione dello sfollamento sondando disponibilità di accoglienza e flussi di spostamento. Il 13 ottobre 1942, come se si aspettasse ciò che sarebbe accaduto di lì a poco, la Dgspa pose finalmente le basi per un nuovo "piano di sfollamento" inviando ai prefetti una circolare nella quale chiedeva di valutare la capacità ricettiva della provincia e di elaborare due progetti: uno di sfollamento interno al territorio provinciale, per coloro che erano o sarebbero rimasti senza casa (Progetto per la sistemazione della popolazione priva di alloggio per effetto dei bombardamenti aerei); uno per l'assorbimento di sfollati provenienti da altre provincie e regioni (Progetto di assorbimento di sfollati da altre zone)2.

Nella notte tra il 22 e il 23 ottobre, 100 aerei del Bomber Command raggiunsero il cielo di Genova. Alcuni spezzoni incendiari e bombe colpirono anche Torino. Era l'inizio di una grande e violentissima offensiva aerea sull'Italia e, conseguentemente, di una nuova fase nel fenomeno dello sfollamento. Da quell'ottobre 1942, infatti, la quantità e la gravità dei bombardamenti fecero esplodere quello che le autorità fasciste chiamarono "sfollamento di massa": centinaia di migliaia di italiani iniziarono ad allontanarsi dalle grandi città e dai maggiori nodi industriali e portuali distribuendosi per lo più nelle immediate periferie, ma anche, esaurita la capacità ricettiva di queste, in territori lontani che avevano spazi e strutture per ospitarli.

Ciò che emerge dai documenti è che la reazione delle autorità e degli uffici centrali fu un intenso e frenetico tentativo - condizionato dal grave ritardo e dall'urgenza - di gestire e regolamentare questo fenomeno, ma, diversamente da ciò che mi ero aspettata conoscendo le gravi carenze e la molta improvvisazione che caratterizzarono altri importanti settori (per esempio quello alimentare), tra l'autunno 1942 e l'estate 1943, sembrano essere stati discretamente efficaci (con inevitabili differenze geografiche dovute alle diverse capacità degli enti locali). Già il primo novembre 1942 furono finalmente emanate nuove direttive riguardanti lo sfollamento che sarebbero entrate in vigore a partire dal 15 novembre. Esse definivano - per la prima volta dalle disposizioni elaborate nel 1939 - alcune precise e articolate procedure burocratiche, allegando anche i moduli necessari per attuarle. Procedure che dovevano servire: a seguire e registrare gli spostamenti 
degli sfollandi e il loro numero; ad accertarsi che essi avessero già individuato un luogo pronto a ospitarli; a risolvere il problema della distribuzione delle tessere annonarie e, soprattutto, delle derrate che dovevano soddisfarle.

Al Partito nazionale fascista (Pnf) non era mai sfuggita l'importanza della sfida che si giocava nel campo dell'assistenza alla popolazione civile e dello sfollamento in particolare ed esso aveva ininterrottamente cercato di avere un ruolo in questi ambiti. Il suo impegno, però, a lungo non era stato preso in considerazione dal Ministero dell'Interno, se non per azioni di stimolo allo sfollamento volontario e per la creazione di punti di aiuto e ristoro presso gli scali ferroviari o lungo $\mathrm{i}$ tragitti dei migranti. Il primo compito effettivamente organizzativo fu affidato al Pnf nel dicembre 1942 ed era collegato all'eventualità di uno sfollamento obbligatorio: se fosse divenuto necessario evacuare gli abitanti delle città maggiori, $\mathrm{i}$ gruppi rionali del partito, poiché più capillarmente distribuiti nel territorio urbano, avrebbero sostituito gli uffici comunali nella consegna dei "fogli di via". Ma questo al Pnf non bastava: voleva avere un ruolo più ampio e incisivo e, soprattutto, di azione autonoma nei compiti assistenziali, anche in quelli più continuativi da gestire nei luoghi di arrivo degli sfollati. Ciò portò a momenti di tensione tra Segreteria del partito e Ministero dell'Interno. Per esempio nel dicembre-gennaio 1942-43, quando Vidussoni (l'allora segretario del Pnf) diramò alcune istruzioni (riguardanti l'alloggiamento degli sfollati) che contrastavano con le direttive ministeriali e il ministro dell'Interno Buffarini Guidi lo richiamò all'ordine rimarcando che «tutta la materia dello sfollamento è demandata alla competenza dei Prefetti e dei Podestà», non del partito. Nel marzo 1943 il Pnf riuscì a ottenere un ruolo istituzionalizzato nell'assistenza ai sinistrati, ma si trattava però, ancora, di «assistenza di primo intervento» rivolta solo a coloro che non avevano più un tetto e da organizzare nelle città bombardate, non nei luoghi di arrivo degli sfollati. La fine del governo Mussolini e l'insediamento del governo Badoglio non videro nuove, significative disposizioni né cambiamenti o interruzioni nel lavoro degli organi coinvolti nel fenomeno dello sfollamento. A modificarsi furono, invece, la tipologia e il flusso di popolazione in movimento a causa della guerra. Infatti agli sfollati per colpa dei bombardamenti si erano uniti, dal luglio 1943, i profughi che salivano a nord spinti dal fronte che avanzava, e, dopo 1'8 settembre, le migliaia di evacuati per ordine tedesco.

Fu con la Rsi che il partito (divenuto Pfr, Partito fascista repubblicano) riuscì finalmente a "mettere le mani" sul sostegno destinato a sfollati, profughi ed evacuati. Il 12 ottobre 1943, infatti, una circolare del segretario del Pfr comunicò alle proprie federazioni provinciali e alle amministrazioni comunali che il lavoro di assistenza ordinaria e di guerra doveva essere trasferito dagli Eca (Enti comu- 
nali di assistenza) agli Ecfa (Enti comunali fascisti d'assistenza), [nei documenti chiamati, a volte, anche Efa (Enti fascisti di assistenza) o Eaf (Enti di assistenza fascista)], nuovi organi che le federazioni del Pfr dovevano creare in ogni comune. La Dgsg (Direzione generale per i servizi di guerra) del Ministero dell'Interno e i capi provincia, però, non vennero completamente estromessi: relativamente all'assistenza di guerra, infatti, era comunque ai capi provincia che sarebbero stati inviati i fondi necessari a garantirla; inoltre gli Ecfa avrebbero dovuto operare seguendo le direttive emanate fino a quel momento dal Ministero dell'Interno. Dall'ottobre 1943 al febbraio 1944 si definì la struttura dell'assistenza fascista: il 12 febbraio 1944 gli Epfa (Enti provinciali di assistenza fascisti) andarono a completare, in posizione intermedia, una piramide che vedeva in cima l'Ente nazionale (Enfa) e ai piedi gli Enti comunali. Ma in merito alle competenze degli Enti provinciali, nei mesi successivi nacquero alcuni problemi tra l'Enfa e la Dgsg, cioè tra partito e Ministero, poiché per quest'ultimo a livello provinciale il referente per tutta la materia doveva essere il capo provincia: egli distribuiva i finanziamenti e quindi a lui gli Ecfa dovevano rendere conto. L'esperienza degli Ecfa fu fallimentare. Le cause furono, probabilmente, la ridondante struttura piramidale degli uffici fascisti, l'inesperienza nella gestione dell'ampio e complesso meccanismo dell'assistenza di guerra (aggravata dall'urgenza con la quale gli Ecfa dovettero sostituire gli Eca) e la confusione nella distribuzione delle competenze. Per prime, autonomamente, alcune prefetture, spinte dalla necessità di porre rimedio alle carenze degli Ecfa, tolsero a questi la responsabilità degli sfollati e dei sinistrati e la restituirono ai comuni. Poi fu il Ministero dell'Interno stesso a prendere la medesima decisione (3 luglio 1944).

Nel giro di pochi mesi i problemi legati ai profughi e soprattutto agli evacuati divennero assai più gravi e impellenti di quelli relativi agli sfollati, fino a far passare questi ultimi in secondo piano.

Di tutte e tre le categorie doveva occuparsi la Dgsg, ma la necessità reale e politica di assistere i civili più colpiti dalla guerra fece intervenire direttamente la Presidenza del Consiglio dei ministri la quale, il 20 novembre 1943, creò alle proprie dirette dipendenze l'Enap (Ente nazionale per l'assistenza ai profughi e la tutela degli interessi delle provincie invase), un ente, con diramazioni sul territorio della Rsi simili a quelle delle organizzazioni del Pfr, che doveva occuparsi dell'assistenza agli italiani originari delle regioni conquistate dagli Alleati. Anche la Dgsg, però, si occupava di profughi ed evacuati e la divisione dei ruoli tra i due organi non era del tutto chiara. Sicuramente l'Enap si interessava solo a quelli provenienti dalle terre occupate dal nemico, mentre la Dgsg doveva preoccuparsi anche di tutti coloro che lasciavano, o erano costretti a lasciare, regioni 
ancora della Rsi. Per quanto riguardava l'assistenza economica, comunque, gli ambiti non erano distinti, poiché la Dgsg doveva prendersi cura anche dei profughi dell'Enap. Che ci fossero confusione e anche sovrapposizione di competenze lo si vede bene da un fatto che avvenne nel giugno-luglio 1944. I profughi che si allontanavano volontariamente dalle loro case a volte lo facevano lasciando nella terra d'origine alcuni parenti che sceglievano di rimanere o di partire in un secondo momento. La complessità del viaggio, l'imprevedibilità del luogo in cui sarebbero stati accolti, le difficili comunicazioni postali, telegrafiche e telefoniche spesso rompevano ogni collegamento tra membri della stessa famiglia che erano partiti in tempi diversi. Chi, poi, si era trasferito a nord, lontano dalla propria casa (provvisoriamente per motivi di lavoro o di studio, o stabilmente perché emigrato), prima che la terra d'origine venisse conquistata dagli Alleati, quando ciò avveniva perdeva i contatti coi cari lontani. E se questi si erano messi in cammino per raggiungerlo, difficilmente riusciva a seguirne gli spostamenti. Di fronte a tali situazioni, l'Enap decise di creare degli Uffici ricerche profughi presso le sedi provinciali dell'Ente. Il loro scopo era quello di stabilire il collegamento tra profughi imparentati che si trovavano a risiedere in località diverse del territorio della Rsi. Nello stesso identico giorno (4 giugno 1944) in cui la Direzione generale dell'Enap ordinava alle Commissioni provinciali di creare tali uffici, la Direzione del Pfr prospettava al Ministero dell'Interno l'esigenza di costituire un «Ufficio centrale per la ricerca e per le notizie di sfollati provenienti dalle Provincie invase»». Un primo "incrocio" di competenze è già nel fatto che fosse il Pfr, benché l'ambito non fosse di sua pertinenza, a segnalare al Ministero la necessità di un tale servizio. Il motivo forse risiedeva nel fatto che, come sappiamo, il partito era sempre molto attento alle reazioni psicologiche degli italiani e ai riflessi che esse avevano sul consenso alla Rsi. Il Ministero comunicò la cosa alla Dgsg il 15 luglio e l’Ufficio centrale fu creato.

\section{I fascisti in fuga}

Tra i profughi che risalivano volontariamente la penisola c'erano anche coloro, singole persone o famiglie intere, che erano, o erano stati, in qualche modo coinvolti nelle strutture fasciste. La gestione del loro trasferimento a nord fu totalmente affidata al partito. La prima direttiva che sono riuscita a rintracciare e ad 
analizzare - poiché citata da Giovanni Cipollini - è un telegramma inviato dal segretario del Pfr Alessandro Pavolini ad alcuni capi provincia il 16 maggio 19444. In esso però viene fatto riferimento a «precedenti istruzioni». Come sappiamo, al 16 maggio 1944 il fronte era ancora sulla linea Gustav e attorno ad Anzio, ma cinque giorni prima era iniziata la quarta e ultima battaglia di Montecassino che si concluse il 18 maggio con la vittoria degli Alleati. Il 22-23 maggio avvenne lo sfondamento del fronte ad Anzio. Il telegramma di Pavolini fu inviato ai capi delle provincie di Ancona, L'Aquila, Arezzo, Ascoli Piceno, Firenze, Grosseto, La Spezia, Livorno, Lucca, Macerata, Pesaro, Pisa, Pistoia, Rieti, Siena e Terni. Era rivolto, quindi, a quasi tutti i territori provinciali compresi tra la linea Gustav e la linea Gotica. Durante la mia ricerca sul fenomeno dello sfollamento nella provincia di Forlì (territorio che si trovava al di sopra della linea Gotica) tra $\mathrm{i}$ documenti conservati nell'Archivio del Gabinetto della Prefettura forlivese ho trovato un telegramma di Pavolini identico a quello del 16 maggio giunto al capo provincia il 30 giugno 1944, quando il fronte si trovava tra Ascoli Piceno e Ancona $^{5}$. Suppongo, quindi, che con l'avvicinarsi del nemico alla Gotica siano state coinvolte dalle direttive riguardanti lo sfollamento dei fascisti anche le provincie subito a nord di essa.

In base alle disposizioni contenute nel telegramma del 16 maggio ogni commissario federale del partito doveva affidare la gestione dello sfollamento delle famiglie fasciste a una persona di fiducia la quale, oltre a organizzare la partenza, doveva accompagnare gli sfollandi nel loro viaggio. La prima destinazione sarebbe stata Bologna, dove, presso la Federazione provinciale del Pfr, stava attivandosi un apposito "Ufficio tappa e smistamento". Da quel punto sarebbe stato tale ufficio a prendersi cura dei profughi accogliendoli e assistendoli per il tempo necessario, consegnando a ogni famiglia un documento con elencati i nomi e i dati delle persone in viaggio e, infine, smistando le famiglie stesse verso le provincie di destinazione, oppure inviandole all' "Ufficio secondario tappa e smistamento" allestito presso la Federazione di Brescia. I fiduciari delle provincie di partenza, esaurito il loro compito, dovevano rimanere a disposizione della Direzione del partito presso la Federazione di Brescia.

Telegramma n. 3270 inviato da Pavolini ai capi delle provincie di Ancona, L'Aquila, Arezzo, Ascoli Piceno, Firenze, Grosseto, La Spezia, Livorno, Lucca, Macerata, Pesaro, Pisa, Pistoia, Rieti, Siena, Terni, oggetto: Disposizioni per lo sfollamento al Nord delle famiglie dei fascisti, 16.05.1944. Citato in Cipollini 1989. 
Una circolare di Pavolini dell'11 giugno dava ulteriori istruzioni: in caso di «imminente invasione o sgombero delle Provincie» gli iscritti al Pfr dovevano essere suddivisi in tre gruppi

a) Fascisti che per essere particolarmente conosciuti o comunque perché volontariamente scelgano questa soluzione debbono trasferirsi al Nord. Per costoro, curare prima e tempestivamente l'invio al Nord delle rispettive famiglie. Per la destinazione di esse, un tuo incaricato può prender contatto con la Direzione del Partito la quale curerà la scelta di località la più adatta possibile. Attraverso tale incaricato il Partito curerà inoltre l'assistenza per tali famiglie, con sussidi giornalieri, acconti, ecc. Quanto ai fascisti di tali gruppi, essi rimarranno sul posto fino all'ultimo momento, ritirandosi insieme ai dirigenti designati all'uopo. Occorre perciò tenere pronti ed eventualmente nascosti gli automezzi ed altri veicoli necessari, nonché scorte di carburanti.

b) Massa dei fascisti. Meno noti o che comunque vogliano o debbano restare in provincia proponendosi di «mimetizzarsi» con l'ambiente, eventualmente anche attraverso provvisorio spostamento di domicilio locale, ecc. Tali fascisti debbono avere la consegna di alimentare localmente un fascismo clandestino, simile nelle sue estrinsecazioni a quello che è l'attività dei partiti clandestini nostri avversari o comunque dei nostri oppositori in genere nelle provincie da noi controllate.

c) Nuclei di attivisti. Elementi scelti e particolarmente idonei che accettino di costituire bande, di dare vita a un ribellismo fascista od anche - in accordo con le autorità militari germaniche - si mettano a disposizione per attentati terroristici, radio clandestine, ecc. ${ }^{6}$.

Nella documentazione relativa allo sfollamento e ai vari aspetti a esso connessi è soprattutto del primo gruppo che troviamo notizie ed è su di esso che mi concentro qui. Per i fascisti, quindi, lo sfollamento era volontario. L'unica regola da seguire era che per primi dovevano partire i famigliari di coloro che erano più compromessi e solo in un secondo momento questi ultimi. Della destinazione doveva occuparsi colui che era stato incaricato dal commissario federale, se necessario chiedendo aiuto alla Direzione del partito. Probabilmente, quindi, le province di accoglienza finale vennero definite man mano che procedeva lo sfollamento, senza che esistesse un progetto.

I pochi casi di sfollamento di gruppi di fascisti sui quali ho informazioni più precise fanno emergere che furono seguiti anche vari altri canali, oltre a quello 
dell'aiuto chiesto alla Direzione del Pfr, in particolare: accordi tra federazioni del partito e scelte del tutto arbitrarie di singole federazioni.

Un caso esemplificativo riguarda le famiglie fasciste della provincia di Perugia. Il 7 giugno 1944 il capoprovincia, Rocchi, scrisse questo telegramma a Buffarini Guidi:

Sfollamento famigliari dei fascisti compromessi di questa provincia soggetti sicurissime rappresaglie caso invasione et aggirantesi circa quattromila unità est impossibilitato per assoluta mancanza automezzi locali requisiti autorità germaniche per esigenze belliche alt Ministero intesa Direttorio Nazionale Partito provveda immediato invio maggior numero possibile automezzi non requisiti autorità germaniche esigenze belliche per procedere detto sfollamento nord Italia località già predisposta questa Federazione alt Prego assicurare alt?

Il 20 giugno un altro telegramma di Rocchi, inviato dalla provincia di Forlì, comunicava:

Lasciato Perugia sera 16 insieme Comandi Tedeschi dopo avere sfollato tutte famiglie fasciste che hanno chiesto allontanarsi. Numero totale sfollati 600 di cui 400 sono ancora Forlì attesa raggiungere definitiva sistemazione ${ }^{8}$.

Non è chiaro quale fine avessero fatto gli altri circa 3.400 fascisti che dovevano sfollare da Perugia. Le parole di Rocchi possono essere interpretate in due modi: o egli aveva scritto 4.000 sperando di ottenere i mezzi di trasporto almeno per 600 , oppure quei 600 erano complessivamente coloro che, alla fine, erano realmente sfollati.

Tre giorni dopo, il 23 giugno, era il capo provincia di Forlì a scrivere al Gabinetto del Ministero dell'Interno a nome di Rocchi:

Prefetto Rocchi chiede autorizzazione prelievo da deposito Cesena litri mille benzina che dovrebbero servire per inoltro suoi 500 profughi provenienti da Perugia et che da giorni sostano a Forlì. Dette unità dovrebbero raggiungere Novara?.

Telegramma n. 5104 inviato dal capo provincia di Perugia Rocchi al Ministro dell'Interno Guido Buffarini Guidi il 07.06.1944, ACS, MI, DGSG, Ag, b. 6.

Telegramma senza numero scritto dal capo provincia di Perugia, da Forlì, a Buffarini Guidi il 20.06.1944, ACS, MI, DGSG, AG, b. 6. II 17 giugno il fronte era sulla linea Grosseto-Ascoli Piceno (Grosseto era già stata liberata, Ascoli Piceno no), a pochissima distanza da Perugia.

Telegramma n. 2488 scritto dal capo provincia di Forlì, a nome del capo provincia di Perugia, al Gabinetto del MI il 23.06.1944, ACS, MI, DGSG, AG, b. 6. Ė evidente la non corrispondenza delle cifre riguardanti il numero di fascisti fermi a Forlì. 
Che ci fosse improvvisazione lo rivela il caso della provincia di Bergamo, dove l'arrivo di famiglie fasciste non era previsto. Il 17 giugno, infatti, il capo provincia così scrisse al Gabinetto del Ministero dell'Interno:

In questi giorni affluiscono questa Provincia numerose famiglie sfollate et fasciste da Provincie Marche e Toscana. Poiché tale afflusso non est stato precedentemente segnalato et comunque determina impossibilità sistemazione et notevoli disagi servizi vari et alimentari. Prego vivamente disporre perché siano impartite opportune disposizioni per prevenire et disciplinare tali movimenti $[\ldots]^{10}$.

Cinque giorni dopo il ministro in persona rispose al capo provincia di Bergamo ordinandogli di accogliere «senza eccezione» gli sfollati che avevano già raggiunto la sua provincia e quelli che sarebbero arrivati"

\section{Evacuazioni e centri di raccolta lungo la penisola}

A rendere particolarmente drammatici e caotici i mesi dopo l'8 settembre 1943 furono soprattutto le massicce evacuazioni forzate ordinate dai comandi tedeschi, sia in prossimità delle linee di difesa man mano allestite all'interno della penisola, sia lungo entrambe le coste, tirrenica e adriatica, per tutta la loro lunghezza [Cortesi 2011]. A creare i problemi più gravi fu il fatto che, nella maggior parte dei casi, i tedeschi imponevano gli sgomberi senza preavvertire le autorità centrali della Rsi e interagendo direttamente con le prefetture. Erano queste ultime che, con tono disperato, davano notizia degli ordini di evacuazione al Ministero dell'Interno descrivendone le conseguenze drammatiche e chiedendo aiuto. Il ministero cercò ripetutamente, soprattutto tra il novembre 1943 e il maggio 1944, di convincere i tedeschi a non ordinare ulteriori evacuazioni, ma, inascoltato, non poté fare altro che tentare di "accompagnare" gli evacuati a nord gestendone il flusso, cercando spazi nei quali sistemarli e racimolando risorse con cui sfamarli. Fin dai primi sgomberi i tedeschi ordinarono alle autorità italiane di creare dei luoghi nei quali raccogliere e assistere provvisoriamente gli evacuati prima del loro trasferimento a nord. Su questi luoghi, chiamati "centri di raccolta", sono riuscita a trovare alcune informazioni importanti, anche se non è possibile dire con esattezza quanti siano stati né, quindi, l'ubicazione di tutti. Di quelli noti, inoltre,

Telegramma n. 1558 inviato dal capo provincia di Bergamo al Gabinetto del Ml il 17.06.1944, Acs, MI, Dgsg, Ag, b. 6. Non è chiaro se il capo provincia intenda "numerose famiglie di sfollati e numerose famiglie fasciste", oppure "numerose famiglie fasciste sfollate", ma questo non inficia la mia analisi.

11 Cfr. Telegramma n. 3457 inviato da Buffarini Guidi al capo provincia di Bergamo il 22 giugno 1944, Acs, MI, Dgsg, Ag, b. 6 . 
non conosciamo le date di creazione e successivo abbandono. Neppure sappiamo esattamente: quanti italiani ciascuno di essi accolse e se siano stati solo evacuati o anche sfollati e profughi volontari; se ospitarono solo coloro che si spostavano a nord o anche chi era destinato al lavoro in Germania. Negli archivi centrali della Rsi c'è pochissimo materiale su essi. Probabilmente più ricchi di notizie sono gli archivi periferici e, sicuramente, lo è la memoria popolare (sia quella di coloro che vi sostarono, sia quella di coloro che risiedevano nelle vicinanze) così come dimostrano, per esempio, alcune testimonianze raccolte da Gabriella Gribaudi e articoli commemorativi comparsi su giornali locali nel dopoguerra [Gribaudi 2005, 480, 483-484; Isabelli 2003]. Ho scelto di chiamarli “centri”, come li denominavano le autorità tedesche e italiane di allora, e non "campi", come troviamo in alcune testimonianze e ricostruzioni fatte oggi, poiché questo secondo termine dà inevitabilmente a essi una connotazione negativa, mentre vorrei mantenere un linguaggio asettico, più burocratico. Ma che la vita in essi sia stata molto simile a quella in veri e propri lager, così come raccontano i testimoni, lo rivelano anche gli efficienti e freddi documenti degli uffici fascisti.

Da una visione d'insieme delle fonti a disposizione sono giunta a ritenere che fossero i tedeschi a decidere se e dove servisse un centro di raccolta. In genere nei luoghi prescelti era già presente una loro guarnigione folta ed efficace, o un contingente utilizzabile di forze dell'ordine italiane. Alcuni centri nacquero con la funzione di collettori degli evacuati prima radunati in punti di raccolta minori. Di questi ultimi, alcuni furono pensati e organizzati per tempo, altri furono improvvisati a seconda delle necessità. Per i centri principali o di media importanza furono coinvolte le autorità centrali dello Stato fascista, per gli altri i tedeschi si rivolsero direttamente alle autorità locali; credo che a volte gli accordi siano stati presi direttamente con le amministrazioni comunali, saltando, cioè, anche la figura del capo provincia. In queste pagine accennerò solo ai centri più importanti. I primi dei quali abbiamo notizia sono quelli creati nelle zone di Frosinone, Littoria e Roma.

Nel territorio di Frosinone pare che ce ne siano stati due molto importanti, uno a Ceprano e uno a Ferentino, funzionanti contemporaneamente fino al 25 novembre 1943. Ceprano si trova tra Cassino e Frosinone, quindi era subito alle spalle della linea Gustav. Ferentino è tra Frosinone e Anagni, quindi più a nord. In genere gli evacuati raccolti a Ceprano venivano poi spostati a Ferentino. Pare che fin dal 26 luglio 1943 a Ferentino si fosse insediata una piccola guarnigione tedesca, inserita nella Divisione corazzata "Hermann Göring". Fu alla fine di questo stesso ottobre che le autorità fasciste e tedesche istituirono il centro di raccolta e smistamento degli evacuati provenienti da territori in prossimità del fronte di Cassino 
utilizzando la casa della Divina Provvidenza e il collegio Martino Filetico. Dalle varie fonti analizzate appare che quello di Ferentino sia diventato il centro più importante di tutta la zona, provincia di Littoria compresa. Il 25 novembre venne soppresso il centro di Ceprano e le oltre 20.000 persone lì radunate, più altre 10.000 evacuate in quei giorni, furono condotte a Ferentino in attesa di essere destinate al luogo di raccolta creato presso la sede dell'industria Breda a Roma, e da lì partire per località più a nord. Nel gennaio successivo il centro di Ferentino era ancora attivo. Il 17 marzo 1944 un nuovo bombardamento aereo alleato spinse gli abitanti ad abbandonare il paese, ma pare che il centro per sfollati abbia continuato a funzionare.

I centri di raccolta più grandi e importanti alle spalle della linea Gustav, quelli nei quali venivano riversati tutti gli evacuati prima radunati nei centri minori, furono quelli creati attorno a Roma. Le strutture da utilizzare vennero selezionate dal locale comando tedesco il quale chiese alle autorità locali italiane (in particolare al capo provincia di Roma) i necessari lavori di adattamento ${ }^{12}$. I luoghi scelti furono sette: l'ex centro chimico militare di Cesano, gli stabilimenti della Breda (in via Casilina), la caserma in piazza Santa Croce in Gerusalemme (chiamata più brevemente "caserma Santa Croce"), Forte Braschi, Forte Aurelio, la caserma Lamarmora in piazza San Francesco a Ripa e l'Istituto San Michele a Ripa Grande. A queste sembra che sia stato poi aggiunto, nella primavera 1944, l'Albergo di Russia ${ }^{13}$. Il centro creato alla Breda pare essere già funzionante alla fine del novembre $1943^{14}$. Probabilmente fu il primo a essere allestito, poi, nel gennaiofebbraio 1944, a causa dell'afflusso sempre più massiccio di evacuati, d'accordo con le autorità tedesche, il capo provincia incaricò il governatore di Roma «di procedere nel più breve tempo all'adattamento di alcuni fabbricati ad accantonamenti per gli sfollati provenienti dalle regioni evacuate al sud di Roma» ${ }^{15}$. Il centro di raccolta di Narni (provincia di Terni) fu creato tra il 29 marzo e il 7 aprile 1944. Che il 29 marzo ancora non esistesse lo capiamo da una comunicazione inviata dal capo provincia di Roma al Ministero dell'Interno relativa al sovraffollamento dei centri della Breda e di Cesano e alla grave situazione nella

Cfr. Comunicazione n. 01 del capo provincia di Roma al Gabinetto del MI del 03.04.1944, Acs, MI, Dgsg, Ag, b. 6.

Cfr. Relazione sui lavori in corso di esecuzione per la sistemazione dei Centri raccolta sfollati, scritta dal direttore dei lavori il 31.03.1944 e poi da questi consegnata al capo provincia, Acs, Ml, Dgsg, Ag, b. 6.

14 Cfr. Cronologia dell'Anpi laziale nel sito www.storiaxxisecolo.it/cronologia/cronoloreslazio/cronoreslaz.html.

Relazione sui lavori in corso di esecuzione per la sistemazione dei Centri raccolta sfollati, scritta dal direttore dei lavori il 31.03.1944, cit. 
quale si trovavano coloro che erano in essi raccolti. Sottolineando «la necessità di trasferire i predetti sfollati al nord», aggiungeva:

Naturalmente occorrerebbe preliminarmente trovare nell'Umbria, nelle Marche $\mathrm{o}$ in Toscana località adatte per costruirvi centri di sfollamento $[\ldots]$ tali centri, come fu fatto per quelli della Breda e di Cesano, dovrebbero essere predisposti dall'Autorità Germanica, facendosi intendere che essi dovrebbero rappresentare [... incomprensibile] di sosta per un successivo conveniente avviamento verso nord $[\ldots]^{16}$.

Il 7 aprile 1944 il «Centro per raccolta e assistenza sfollati in transito» di Narni era già in funzione, ma, secondo l'Ufficio collegamento con le autorità militari germaniche, aveva una «capacità assai ridotta» e il capo della provincia di Terni aveva scritto un accorato telegramma al suddetto Ufficio, da questo inoltrato, nello stesso giorno, al Comando superiore tedesco: «Contrariamente accordi intervenuti Comandi Tedeschi hanno scaricato in Comune Narni oltre tremila sfollati Comuni Cassino Velletri Cisterna alt Accompagnatori tedeschi hanno preannunciato ulteriori arrivi numerosi sfollati alt $[\ldots]\rangle^{17}$. Comunque, se la capacità di assorbimento del centro di Narni era insufficiente rispetto alle necessità, di certo non era piccola dato che il 12 aprile ospitava ben 8.000 (forse più) evacuati' ${ }^{18}$. Quello di Narni era sicuramente il centro di raccolta più importante nell'Italia centrale a nord di Roma. Altri minori vennero creati verso la fine del marzo 1944 in provincia di Perugia.

Sembra che nella seconda metà dell'aprile 1944 esistessero già punti di raccolta provvisori nei territori di Firenze, ma l'unico documento che ho trovato relativo a una vera e propria struttura appositamente attrezzata in questa zona è dell' 8 maggio e riguarda un centro ancora da creare proprio a Firenze ${ }^{19}$.

\footnotetext{
16 Comunicazione n. 4589 inviata dal capo provincia di Roma al Ml il 29.03.1944, Acs, Ml, Dgsg, Ag, b. 6.

17 Questo telegramma (il n. 2571) scritto dal capo provincia di Terni all'Ucamg (Ufficio collegamento con le autorità militari germaniche) è interamente riportato nella comunicazione senza numero inviata il 07.04.1944 dall'Ucamg al Comando militare superiore germanico e p.c. al Gabinetto del Ml e all'ufficio romano della Dgsg, Acs, Ml, Dgsg, Ag, b. 6.

18 Comunicazione n. 40/C/2068 inviata il 12.04.1944 dall'Ucamg al capo provincia di Terni e p.c. al capo provincia Firenze e alla Dgsg, Acs, MI, Dgsg, Ag, b. 6.

19 Bozza del telegramma inviato dalla Dgsg al capo provincia di Firenze il 08.05.1944, Acs, Ml, Dgsg, Ag, b. 6.
} 


\section{La costruzione di baraccamenti}

Tutti gli evacuati convogliati nei centri di raccolta dovevano poi, o avrebbero dovuto, raggiungere luoghi di accoglienza definitiva nell'Italia settentrionale. Il Gabinetto del Ministero dell'Interno e la Dgsg si trovarono a dover affrontare con urgenza il problema di reperire un luogo dove fermarsi per questa massa di persone. La cosa si fece ancora più drammatica con lo sfondamento della linea Gustav. I tedeschi, infatti, pur dovendo indietreggiare velocemente, non rinunciarono a spingere a nord la popolazione: per primi gli evacuati raccolti negli appositi centri, ai quali unirono i tantissimi che fecero sfollare lungo il cammino. Questo senza preoccuparsi molto del problema di trovare a essi una sistemazione; problema che fu totalmente scaricato sulle spalle della Rsi. Il 9 giugno Buffarini Guidi e il Gabinetto del suo ministero, con la speranza di riuscire a trovare o a creare altro "posto" inviarono a tutti i capi delle provincie a nord della linea Gotica due distinti telegrammi. Molto stringato quello di Buffarini:

Date necessità momento coloro che hanno comunque abitazione hanno supremo dovere imporsi possibili limiti et quindi mettere disposizione sfollati [è da intendersi: sfollati, profughi ed evacuati] vani non strettamente indispensabili propria famiglia; in una parola est necessario comprensione popolazione in modo ricavarne maggior numero possibile vani [Maggiorani 1995, 375-376, nota 44].

Il Gabinetto ordinava di recuperare o creare posto per evacuati, profughi e sfollati in tre modi: cercare abitazioni e vani di vario tipo ancora disponibili, considerando anche ambienti non indispensabili alla vita famigliare all'interno di case private abitate; riparare, con i mezzi e i materiali disponibili sul posto, le case non gravemente danneggiate dai bombardamenti; costruire baracche utilizzando «materiali di risulta da distruzione edifici». Inoltre, entro il 20 giugno i capi provincia dovevano comunicare:

a) numero sfollati [è da intendersi tutte le tre categorie: sfollati, profughi ed evacuati] già sistemati codesta provincia $b$ ) numero vani ottenibili mediante riparazione con mezzi sul posto di case danneggiate et numero sfollati che si potranno alloggiare c) numero sfollati che potranno trovare ricovero in baracche da costruirsi con materiale di risulta indicando fabbisogno legname strettamente necessario ${ }^{20}$.

Circolare telegrafica n. 3195 diramata dal Gabinetto del Ml ai capi provincia il 09.06.194, non ho trovato l'originale ma la sua completa trascrizione nella comunicazione n. 1.8546 inviata dalla Dgsg al capo provincia di Ferrara il 03.09.1944, Acs, Ml, Dgsg, Ag, b. 6. 
Le risposte dei capi provincia ${ }^{21}$ relative al punto a) permisero alla Dgsg di comporre una tabella della quale riporto solo le voci relative all'Emilia-Romagna:

\begin{tabular}{|l|l|l|}
\hline Provincia & n.abitanti & $\begin{array}{l}\text { sfollati } \\
\text { accolti }\end{array}$ \\
\hline Bologna & 683.032 & 92.948 \\
\hline Ferrara & 366.611 & 28.271 \\
\hline Forlì & 422.831 & 30.500 \\
\hline Modena & 448.429 & 36.723 \\
\hline Parma & 373.695 & 140.000 \\
\hline Piacenza & 290.445 & 8.900 \\
\hline Ravenna & 272.500 & 14.505 \\
\hline Reggio Emilia & 360.909 & 37.007 \\
\hline
\end{tabular}

Statistica degli sfollati accolti in alcune provincie italiane a tutto maggio $1944^{22}$.

Per quanto riguarda gli altri dati richiesti, gran parte delle prefetture scrissero frasi come «possibilità riparazione case danneggiate minima» oppure «pressoché nulla per mancanza materiale», e anche «possibilità costruzione baracche pressoché nulla per mancanza totale materiali necessari». Molte si limitarono a un lapidario «capacità ricettiva nulla». Alcune fecero sapere che qualcosa potevano fare grazie alle macerie provocate dai bombardamenti, o al riutilizzo di spazi industriali abbandonati, oppure a materie prime fornite dai comandi tedeschi locali. Altre informarono il ministero e la Dgsg che, in seguito al telegramma inviato dalla Dgsg a numerosi capi di provincia il 15 aprile $1944^{23}$ avevano avviato la progettazione, o addirittura già la costruzione, di baraccamenti; nella maggioranza dei casi, però, i lavori erano bloccati o procedevano a rilento sempre per la mancanza dei materiali necessari. Nei mesi successivi il Ministero dell'Interno tornò ad assicurare apposite erogazioni per la costruzione di baracche e, di fronte a ogni progetto che giungeva dalle provincie, procedette immediatamente e immanca-

\footnotetext{
21 Le risposte dei capi provincia alla circolare n. 3195 del 09.06.1944 sono conservate in parte nella b. 6 e in parte nei sottofascicoli provinciali della b. 111, f. 370 Baraccamenti per sinistrati e sfollati, entrambe in Acs, MI, Dgsg, Ag.

22 Cfr. Statistica sfollati a tutto maggio 1944, ACS, MI, DGSG, AG, b. 6.

23 «Prego telegrafare a vista numero massimo sfollati retrovie fronte sud che codesta provincia può assorbire dei quali sarà preannunciato successivamente arrivo punto conto su particolare interessamento capo provincia per sistemazione sfollati in parola anche in baracche per cui costruzione può richiedersi apposito fondo punto» [Telegramma n. 2060 inviato dalla Dgsg ai capi delle province di Asti, Alessandria, Aosta, Cuneo, Vercelli, Novara, Varese, Como, Bergamo, Brescia, Pavia, Piacenza, Cremona, Mantova, Verona, Parma, Reggio Emilia, Modena, Vicenza, Padova, Apuania, Lucca, Pistoia, Pisa, Arezzo, Siena, Perugia, firmato da Buffarini Guidi, 15.04.1944, Acs, MI, Dgsg, Ag, b. 6].
} 
bilmente all'approvazione e all'accreditamento dell'intera cifra (o di gran parte di essa) preventivata dalla prefettura. I progetti effettivamente realizzati non furono però tanti poiché, comunque, ottenere il finanziamento non voleva dire risolvere il problema del reperimento delle materie prime ${ }^{24}$.

Per quanto riguarda l'Emilia- Romagna, all'inizio del maggio 1944 il commissario straordinario di Rimini avviò un dialogo col capo provincia proponendo la possibilità di costruire un villaggio di baracche in località Taverna del comune di Montecolombo, sul fiume Conca. Il commissario aveva, infatti, un grosso e urgente problema da risolvere: fin da febbraio i tedeschi avevano avviato l'evacuazione della fascia costiera; fino ad allora gli edifici svuotati e distrutti per esigenze militari erano quelli più vicini alla costa, ma a marzo proprio il capo provincia gli aveva comunicato che:

Secondo gli ordini pervenuti dal Comando Germanico la popolazione della fascia litoranea qui appresso indicata, fino ad una profondità approssimativa di $10 \mathrm{~km}$. dalla costa, dovrà tenersi pronta, in caso eventuale di necessità militari, a sfollare in brevissimo tempo, con preavviso, se possibile, di 24 a 48 ore, che potrebbe però essere anche notevolmente ridotto.

La regione di sfollamento è delimitata dai seguenti confini:

- a sud dal confine della Provincia di Pesaro

- a nord dal confine della Provincia di Ravenna

- ad est dal Mare Adriatico

- ad ovest da una linea approssimativamente distante $10 \mathrm{~km}$. dalla costa.

In tale fascia litoranea, che interessa 18 Comuni, restano assorbiti totalmente i Comuni di Cattolica, Misano A., S. Giovanni in Marignano, Riccione, Cesenatico, Gatteo e S. Mauro.

Restano invece assorbiti parzialmente i Comuni di Rimini, Cesena, Savignano, Santarcangelo, Coriano, Gambettola, Longiano, Saludecio, S. Clemente, Morciano, Montecolombo.

Complessivamente questa regione abbraccia un territorio di circa $480 \mathrm{kmq}$. con una popolazione di 146.579 abitanti (compresi gli sfollati da altre Provincie) che è suscettibile di variazioni, data la continua fluttuazione degli sfollati $[\ldots]^{25}$.

Era necessario, quindi, spiegava il commissario al capo provincia, costruire baraccamenti fuori dalla zona di evacuazione.

24 Tutta la documentazione relativa alla progettazione, finanziamento e costruzione di baraccamenti è conservata in Acs, MI, Dgsg, Ag, b. 111, f. 370 Baraccamenti per sinistrati e sfollati.

25 Piano di sfollamento della fascia costiera inoltrato dal capo provincia di Forlì ai comuni interessati il 31.03.1944, Asfo, Agp b. 390, f. 113. In una cartina allegata a questo documento sono indicati i successivi spostamenti che gli sfollati dalle cinque zone costiere dovevano compiere, fino al loro luogo di destinazione esterno alla zona di operazioni militari. Per tutto ciò che riguarda la storia dello sfollamento e delle evacuazioni in provincia di Forlì si veda: Cortesi 2003. 
Il villaggio di Taverna doveva essere destinato agli «sfollati dalle terre del meridione invase dal nemico ${ }^{26}$, che avevano raggiunto la zona di Riccione e Rimini, per un totale di 800 persone; esso distava $13 \mathrm{Km}$ dalla fascia costiera; la vicinanza del fiume consentiva la disponibilità di ghiaia e sabbia, indispensabili materiali da costruzione; il terreno era di proprietà della Congregazione di carità di Montescudo, perciò facilmente requisibile. Il progetto prevedeva la costruzione di sedici padiglioni a uso dormitori, disposti su quattro file, con all'interno i servizi igienici, un fabbricato per la cucina e il refettorio, due strutture identiche erano destinate una all'accoglienza e agli uffici della direzione, l'altra all'ambulatorio medico

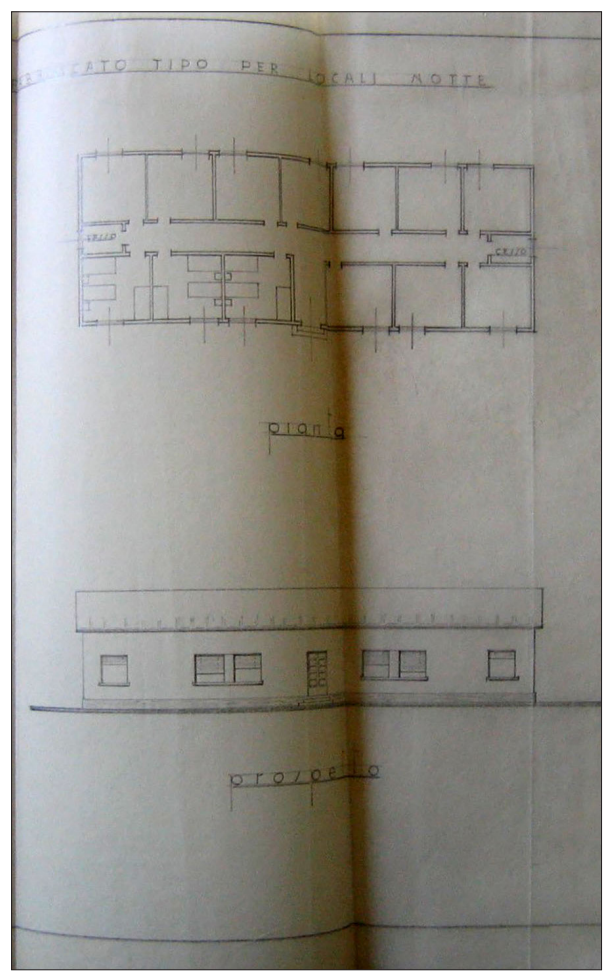

Fig. 1 - Taverna di Montecolombo, dormitorio [ACS, MI, DGSG, AG, b. 111, f. 370, sf. 37033 Forli]. e alla stanza disinfezione; era presente anche un'ampia infermeria con stanze di degenza; il tutto circondato da viali alberati e giardini. A guerra terminata il villaggio sarebbe potuto diventare una colonia climatico-fluviale. «La costruzione è prevista col minimo impiego di ferro cemento e legno [...] soffitti in legno stuoie e gesso ${ }^{27}$. Tenendo conto dei materiali, della manodopera e anche del carburante per i mezzi di trasporto, il preventivo di spesa si aggirava sui 5 milioni e mezzo ${ }^{28}$.

A fine maggio il capo provincia inoltrò al Gabinetto del Ministero dell'Interno tutte le informazioni relative al progetto e ne chiese il finanziamento. Senza sapere che pochi giorni dopo, a fine maggio, il temuto ordine di evacuazione della fascia costiera sarebbe stato effettivamente dato. L'11 giugno il Ministero rispose autorizzando la costruzione del villaggio a Taverna e

\footnotetext{
26 Relazione inviata dal capo provincia di Forlì al Gabinetto del Ml il 25.05.1944 assieme al preventivo e alle planimetrie del villaggio di Taverna, Acs, MI, Dgsg, Ag, b. 111, f. 370, sf. 370-33 Forli. 


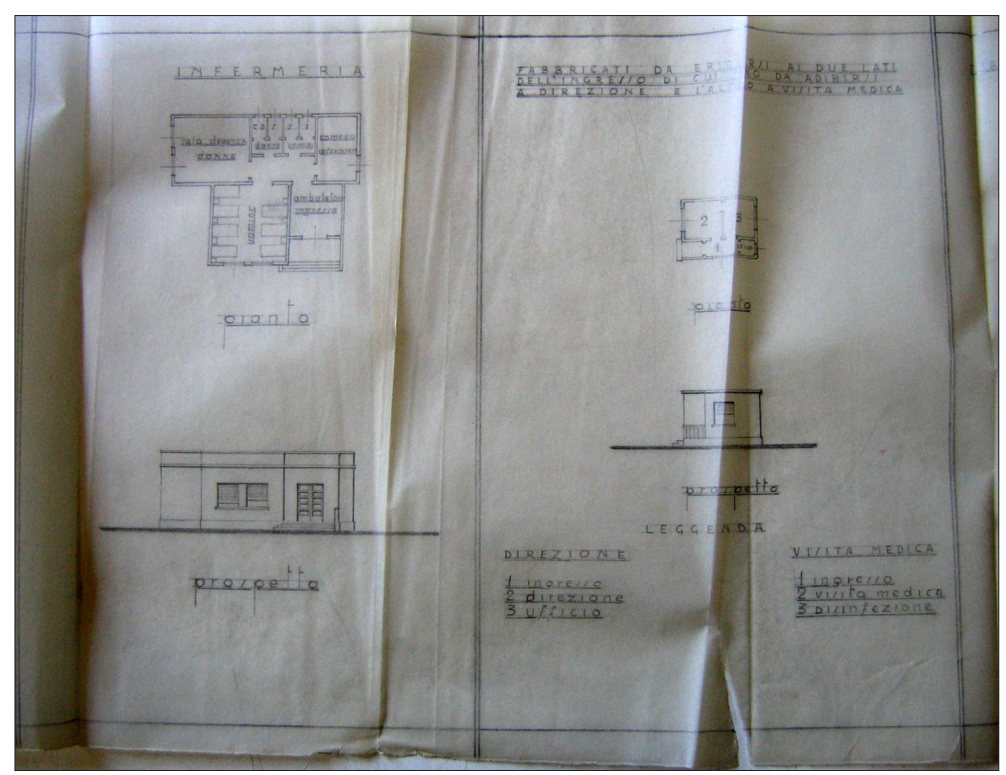

Fig. 2 -

Taverna di

Montecolombo, infermeria, ambulatorio e direzione [ACS, MI, $D G S G, A G, b$. $111, f .370, s f$. 370-33 Forli].

comunicando che in quella stessa data era stato disposto un accreditamento di esattamente 5 milioni e mezzo.

Il problema, a questo punto, era trovare i materiali necessari e farlo in fretta. E non solo per il campo di Taverna. Già il 5 giugno precedente, poco dopo l'ordine di evacuazione, sempre il commissario straordinario di Rimini aveva comunicato al capo provincia che era assolutamente necessario costruire un altro campo di accoglienza, questa volta per i tanti costretti a lasciare le loro case che, per ora, sono stati sistemati, anzi ammassati, in locali antigienici. Il luogo individuato per le baracche era a Corpolò, nelcomune di Rimini.

Tante baracche, nel secondo villaggio sicuramente in numero maggiore che nel primo, e i materiali da costruzione non c'erano. Il 9 giugno arrivò ai capi provincia quel telegramma del Ministero dell'Interno al quale ho già accennato:

Problema sistemazione sfoll. est diventato acuto et deve essere affrontato et comunque risolto al più presto $[\ldots]$

Si è ormai appurato che è impossibile costruire un n. di baracche sufficienti per mancanza materiali necessari, specialmente legno da costruzione.

I capi provincia, devono, con ogni mezzo a disposizione:

1) Ricerca abitazioni e vani ancora disponibili

2) Riparazione di lieve entità di case danneggiate bomb.

3) Costruzione di baracche con materiali di risulta da distruzioni edifici [... $]^{29}$. 
Del villaggio di Corpolò non si parlò più, anche perché per esso non arrivarono finanziamenti, e il capo provincia si attivò per sollecitare i "propri” comuni ad accogliere più evacuati e profughi possibile ${ }^{30}$. Arrivò poi a «requisire due padiglioni in cemento armato della locale ditta Benini, uno dei quali è già stato montato, ed il secondo è ancora da mettere in opera $»^{31}$.

In provincia di Bologna il 31 maggio 1944 l'Eaf (Ente di assistenza fascista) di Imola scrisse al capo provincia chiedendo di finanziare la costruzione di un villaggio per profughi e sfollati costituito da sei fabbricati in muratura, suddivisi in otto stanze, con una capienza di 60 persone ciascuno, una struttura adibita a refettorio e una a servizi igienici.

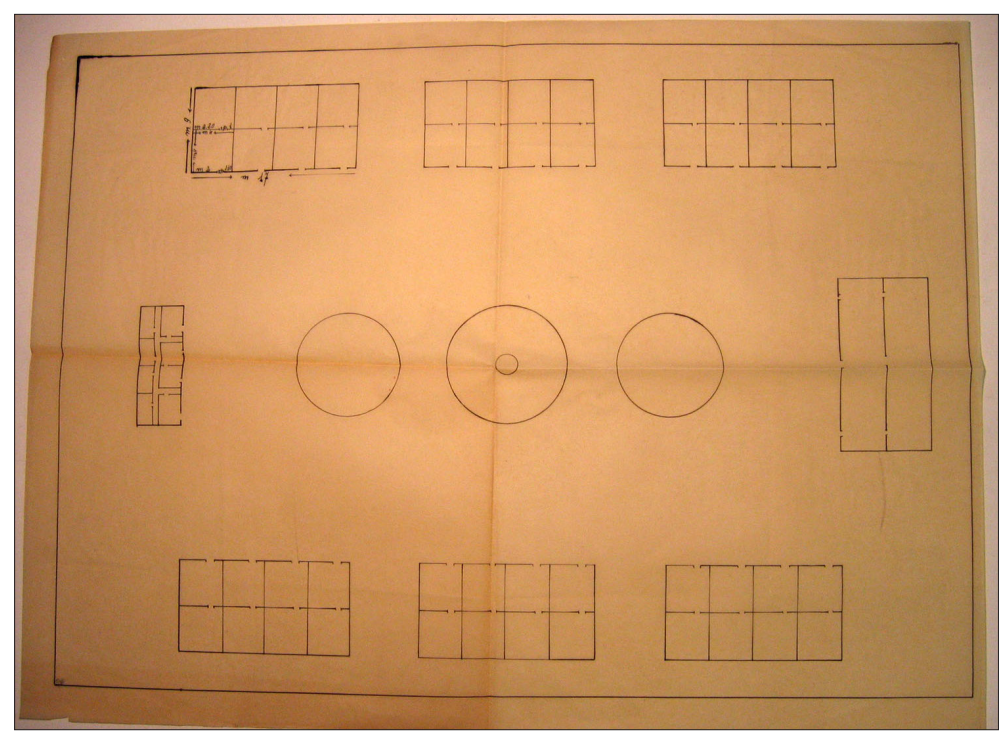

Fig. 3 - Imola, Planimetria villaggio per profughi e sfollati [ACS, $M I, D G S G$, Ag, b. 111, f. 370, sf. 370-14 Bologna].

Il luogo scelto per la creazione della struttura era vicino al fiume Santerno, in un terreno di pertinenza comunale e, da preventivo, la spesa sarebbe stata di circa 2 milioni di lire. L'Eaf prospettava di poter raccogliere 500.000 lire attraverso contributi privati, il resto avrebbe dovuto fornirlo la Dgsg, la quale, il 9 giugno 1944, concesse lo stanziamento ${ }^{32}$.

\footnotetext{
30 Cfr. la Comunicazione inviata dal capo provincia di Forlì alla Dgsg il 29.081944; in questa lettera il capo provincia segnala di avere già informato il ministero di questi sui prowedimenti fin dal 28.06 precedente e poi ancora il 12.07 e il 15.08 . Acs, MI, Dgsg, Ag, b. 111, f. 370, sf. 370-33 Forli.

31 Comunicazione inviata dal capo provincia di Forlì alla Dgsg il 02.09.1944. Acs, Ml, Dgsg, Ag, b. 111, f. 370, sf. 370-33 Forli. La ditta edile Benini aveva iniziato la propria attività nel 1898 e negli a anni '20 era diventata una delle più importanti nell'intera nazione. Costruiva in cemento armato acquedotti, fognature, ponti, capriate, ospedali, insomma tutto quello che veniva a essa richiesto.
}

32 Per tutte queste informazioni si vedano documenti vari in Acs, MI, Dgsg, Ag, b. 111, f. 370, sf. 370-14 Bologna. 


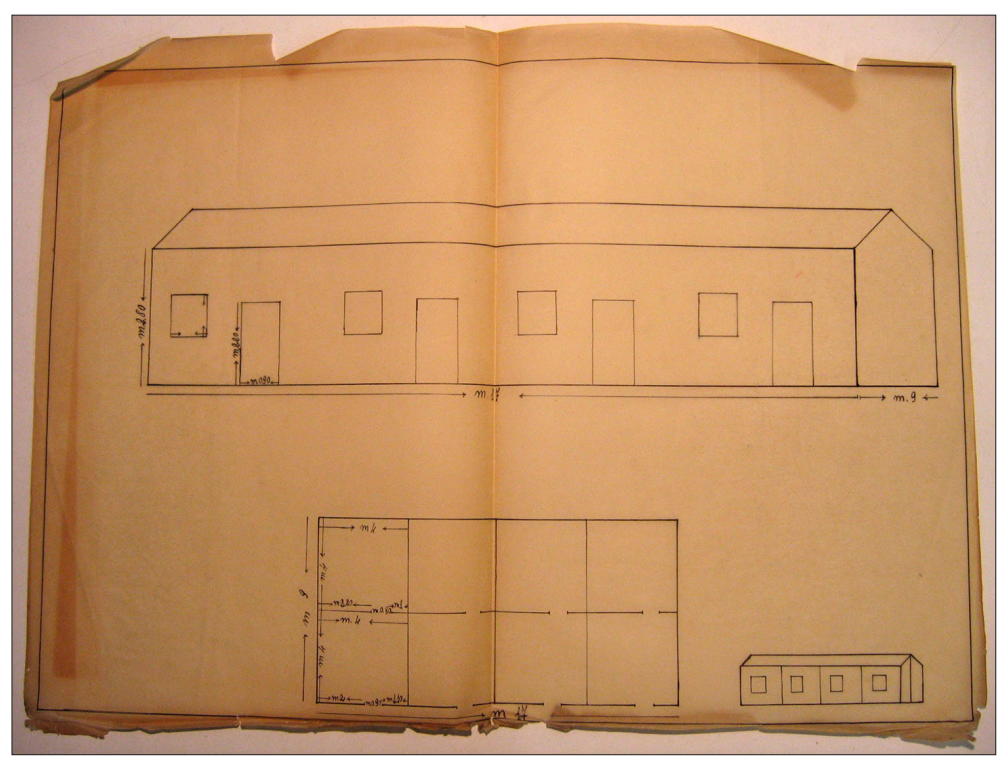

Fig. 4-Imola, Particolare di uno dei fabbricati in muratura adibiti a dormitorio [ACS, $M I, D G S G$, Ag, b. 111, f. 370, sf. 370-14 Bologna].

Relativamente alle altre province emiliane ho notizia di un solo altro villaggio di baracche progettato nel luglio 1944 a Fiorano di Modena e destinato a famiglie costrette a lasciare il capoluogo a causa degli attacchi aerei. Il capo provincia ci tenne a sottolineare che, oltre alle strutture minime indispensabili, cioè dormitori, servizi igienici, cucina e refettorio, erano presenti un pozzo per l'acqua potabile, una lavanderia e la luce elettrica. Spesa preventivata 2.910.000 lire. Il 18 luglio la Dgsg autorizzò i lavori e accreditò alla prefettura tre milioni di lire ${ }^{33}$.

Sembra, invece, che al capo provincia di Parma, Ugo Leonardi, che era anche presidente del Comitato provinciale di protezione antiaerea, non piacesse per niente l'idea di costruire apposite strutture per incursionati o profughi. Nel maggio 1944, infatti, scrisse alla Direzione generale dei servizi di protezione antiaerea una lettera dal tono piuttosto alterato facendo notare come fosse assurdo progettare baraccamenti dato che non c'erano le risorse sufficienti nemmeno per creare pochi, ma necessari rifugi antiaerei:

[...] Allo stato attuale delle cose non è possibile assicurare la costruzione di alcun baraccamento non disponendo del benché minimo quantitativo dei materiali occorrenti. All'uopo si fa notare che l'apprestamento di n. 3 ricoveri, già in stato di avanzata costruzione, si è dovuto sospendere per mancanza di pochi quintali di cemento. [...] Con l'occasione si ritiene doveroso far nuovamente notare e codesto Ministero come il centro urbano di Parma, forte di 80.000 abitanti possiede un numero esiguo di ricoveri convenientemente apprestati, con una disponibilità complessiva di 7.500 posti circa; ciò malgrado codesto Ministero con foglio [...] ha 
creduto affermare che "sembra a questo Ministero sufficiente ai bisogni protettivi della popolazione di codesto Capoluogo il cospicuo complesso di apprestamenti in esso fin qui realizzato". Sta di fatto che questa città ha subito il giorno 25 c.m. il primo violento bombardamento aereo nelle condizioni sopraccennate ${ }^{34}$.

Appena ricevuta, la Direzione di protezione antiaerea, girò l'originale alla Dgsg.

Il presente contributo è una rielaborazione rivista ed ampliata della relazione presentata al convegno "I molti territori della Repubblica fascista. Amministrazione e società nella RSI', Ferrara 27/28 settembre 2017. A fronte dell'impossibilità di sottoporre il testo ad un processo di double blind peer-review, $i$ curatori hanno optato per una revisione preliminare da parte del relativo discussant di sessione.

\section{Bibliografia}

Adorno S. 1986, Lo sfollamento a Pesaro, in Rochat G., Santarelli E. e Sorcinelli P. (eds.) 1986, Linea Gotica 1944. Eserciti, popolazioni, partigiani, Milano: Franco Angeli

Cipollini G. 1989, Il piano di sfollamento totale della provincia di Lucca (maggio-settembre 1944), "Documenti e studi", 8/9

Cortesi E. 2003, L'Odissea degli Sfollati. Il Forlivese, il Riminese e il Cesenate di fronte allo sfollamento di massa, Cesena: Il Ponte Vecchio

Cortesi E. 2011, Sfollati per ordine tedesco, "Storia e problemi contemporanei”, 56

Gagliani D. 1999, Brigate nere. Mussolini e la militarizzazione del Partito fascista repubblicano, Torino: Bollati Boringhieri

Gioannini M. e Massobrio G. 2007, Bombardate l'Italia. Storia della guerra di distruzione aerea 1940-1945, Milano: Rizzoli

Gribaudi G. 2005, Guerra totale. Tra bombe alleate e violenze naziste, Napoli e il fronte meridionale 1940-44, Torino: Bollati Boringhieri

Isabelli 2003, Ricordi di guerra, "Frintinu me...", 3

Legnani M. 1992, Guerra e governo delle risorse. Strategie economiche e soggetti sociali nell'Italia 1940-1943, in Micheletti B. e Poggio P.P. (eds.) 1992, L'Italia in guerra 1940-43, Annali della Fondazione "L. Micheletti”, 5

Maggiorani M. 1995, Uscire dalla città: lo sfollamento, in Dalla Casa B. e Preti A. Preti (eds.) 1995, Bologna in guerra 1940-1945, Milano: Franco Angeli 